\title{
Endoscopic Neuromodulation of Suprascapular Nerve in Chronic Shoulder Pain: A Case Report
}

\author{
Marco Divizia ${ }^{1}$, Giorgio Germani ${ }^{1}$, Ignazio Urti ${ }^{1}$, Farnad Imani $\mathbb{( i D}^{2}$, Giustino Varrassi $\left(\mathbb{D}^{3}{ }^{3}{ }^{*}\right.$ and Stefano \\ Meloncelli ${ }^{1}$ \\ ${ }^{1}$ Valle Giulia Hospital, Roma, Italy \\ ${ }^{2}$ Pain Research Center, Iran University of Medical Sciences, Tehran, Iran \\ ${ }^{3}$ Paolo Procacci Foundation, Roma, Italy \\ "Corresponding author: Paolo Procacci Foundation, Via Tacito 7, Roma, Italy Email: giuvarr@gmail.com
}

Received 2020 April 11; Accepted 2020 April 14.

\begin{abstract}
Shoulder pain is very frequent, especially in middle-aged male adults. Its treatment may be very problematic, mainly in patients who cannot rest and stop their work. At present, it is treated with analgesics, physiotherapy, infiltration of corticosteroids, and/or radiofrequency neuromodulation of the suprascapular nerve. This may be effective but not easy to do. Its efficacy is limited in time, especially because the approach to the nerve trunk may be problematic for its anatomical nature. Ultrasonography has helped, but it is not always completely helpful, due to the small dimension and the complexity of the anatomical structure. In this case report, we describe a more helpful approach to the nerve trunk using an endoscopic technique. The results are very promising. However, larger studies would be necessary to make clear its usefulness.
\end{abstract}

Keywords: Shoulder Pain, Chronic Pain, Neuromodulation, Radiofrequency, NSAIDs

\section{Introduction}

Shoulder pain is a common disease in adults and may become chronic not only due to the difficult detection of a good treatment strategy $(1,2)$, but also because of chronic neuroinflammation as it is observed in any osteoarticular disease. It can be determined by the impingement of articular capsule, disease of the rotator cuff, capsulitis, tendinitis, and degenerative disease $(3,4)$. The management of shoulder pain requires a multimodal approach, including mini-invasive procedures like pulsed radiofrequency (PRF) of the suprascapular nerve, which provides $70 \%$ sensory innervation of the shoulder $(5,6)$. Other techniques like steroid injection and anesthetic blocks have short-term efficacy, while surgical neurectomy causes irreversible paralysis of supraspinatus and infraspinatus muscle.

Pulsed radiofrequency of the suprascapular nerve is a good therapeutic option without damaging motor function, even if the results are not always the same in terms of duration (6-9). This can be due to different factors. First, there are limited published articles with homogeneous populations using the same stimulation parameters. Second, it is not easy to perfectly reach and stimulate small nerves like the suprascapular nerve with a radiofrequency needle. It is quite normal for a pain physician practicing
PRF to have really good results or no pain benefit depending on the millimetric positioning of the needle tip, which often cannot be determined clearly. Ultrasonography has helped to reduce this bias by visualizing the suprascapular nerve and targeting it more clearly (10).

Another problem is related to the anatomic constitution of the suprascapular nerve that is targeted conventionally in the suprascapular notch but is divided into two branches, lateral and medial. They have different functions and supply innervation to the posterior supraspinatus and articular branches to the glenohumeral joint capsule (lateral) while the medial trunk provides motor innervation to the anterior region. This anatomical differentiation makes it important to preserve motor function by isolating and stimulating only the lateral part of the nerve.

As said before, ultrasonography may represent good support. Still, the suprascapular nerve is not very easy to be seen, and thus, the problem of correct PRF neuromodulation of the nerve still exists. For this reason, we tried an endoscopic approach to the suprascapular nerve to have an extremely clear vision of the anatomical structures, identify the neural trunk, and be sure of the stimulation field. 


\section{Case Presentation}

The patient was a 63-year-old man, $181 \mathrm{~cm}$ tall, and 87 kg weight. Since he was 55-years-old, he had arterial hypertension on treatment. No other chronic or neurological pathologies or previous surgical treatments were reported. He had no allergic history to local anesthetics and no coagulation test abnormalities. His shoulder was painful for more than six months, with a limited range of motion of the arm. The pain was reported to the upper and anterior part of the shoulder, radiating to the biceps. He had 24-h pain exacerbated by the movements of internal and external rotation of the arm, with a very painful spotted area localized in the suprascapular notch zone.

The MRI exam had shown minor subscapularis insertional tendinopathy and acromioclavicular joint hypertrophic osteoarthropathy. The orthopedic surgeon had excluded surgical indications, and suggested physiotherapy, without benefits. The prescribed pharmacological treatment consisted of ibuprofen and acetaminophen daily, with limited results. He had received three intra-articular corticosteroid infiltrations, completely useless. A diagnostic suprascapular nerve block with $3 \mathrm{~mL}$ of lidocaine $2 \%$ using ultrasonography had shown the complete remission of pain for six hours.

We decided to perform radiofrequency neuromodulation with an endoscopic approach. The patient received local anesthesia with mild sedation (10 mL of mepivacaine $2 \%$ injected in the surgical site and continuous iv. infusion of propofol $1.5 \mathrm{mg} / \mathrm{kg} / \mathrm{h}$ ). The procedure was performed using Joimax ${ }^{\circledR}$ endoscopic cannula, with small skin incision and continuous washing using normal saline to perform endoscopy. The point of entrance of the endoscope was identified $3 \mathrm{~cm}$ below the suprascapular notch to have plane access to minimize the risk of pleural damage. The visualization of the suprascapular nerve was good, and it was possible to have a clear vision of the nerve path through bone structures. The positioning of the stimulation probe was also clear. The stimulation probe was represented by an electro catheter (VoJager, Alfamed) for suprascapular nerve pulsed stimulation, $40 \mathrm{~cm}$ long, with a $1 \mathrm{~cm}$ tip stimulating terminal. The catheter is flexible with a rigid metallic guide that was removed before stimulation. The stimulation setting was pulsed radiofrequency (Baylis Medical Co.) as 20-millisecond stimulation, 480-millisecond pause, eight minutes total, $42^{\circ} \mathrm{C}$ temperature, and 45 Volt impulse power. Even if the nerve was under our eyes, we used electro-stimulation to be sure. We looked after a radiating pain sensation in the anterior part of the shoulder. The test was considered good when radiating pain was generated at $0.3 \mathrm{~mA}$ (Figures 1-3).

The monitoring of pain was made using the Visual

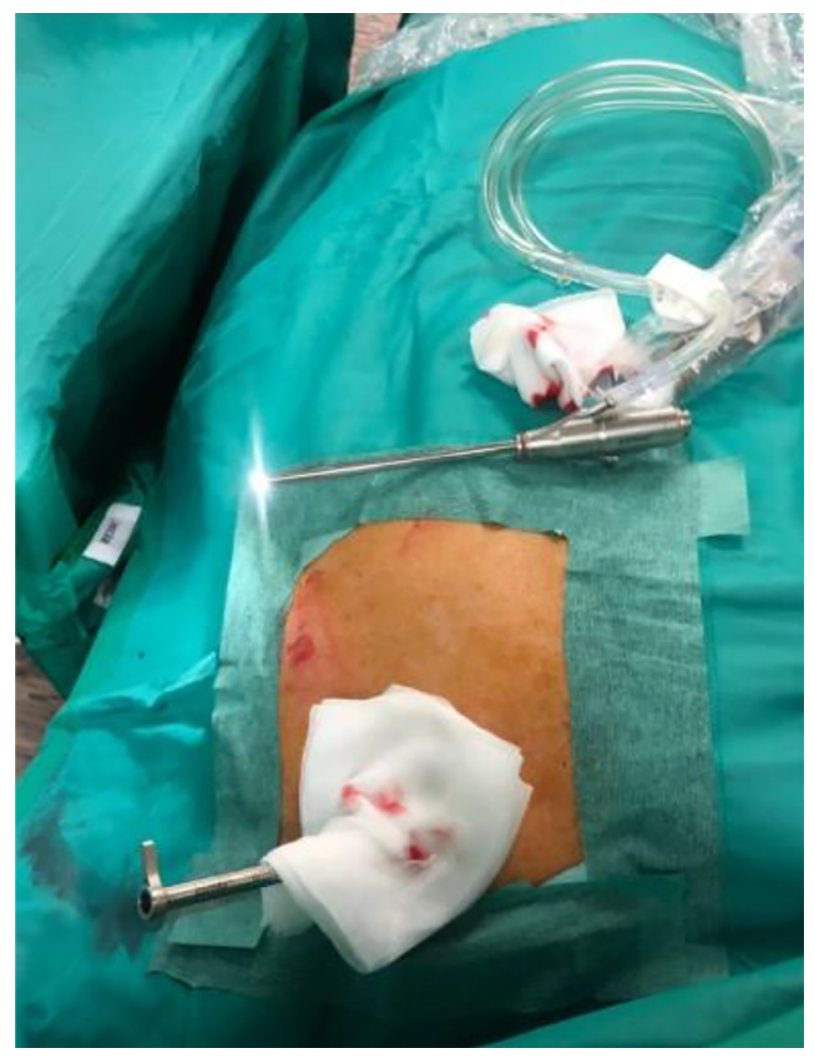

Figure 1. View of the endoscope designed for endoscopic peripherical nerve stimulation. The working cannula is inserted in the suprascapular notch.

Analog scale (VAS = 1 - 10) during resting, movement, and sleep, before and six times after the procedure (two weeks, one month, three months, six months, 12 months, and 18 months), as shown in Table 1. At the same time, the Shoulder Pain and Disability index (SPADI) was determined. The SPADI is a 13-item questionnaire to assess pain (five items $=0-50$ points) and disability (eight items $=0-80$ points) related to shoulder problems (Table 2).

The procedure lasted $45 \mathrm{~min}$. It was conducted without side effects. The patient was discharged from the hospital $24 \mathrm{~h}$ after the surgery. The results of VAS and SPADI are summarized in Table 1 and Table 2, respectively. The reduction of VAS at rest (from 5 to 3 ), during movement (from 9 to 4 ), and at night (from 8 to 3 ) during the whole follow-up period made the patient satisfied. Also, the SPADI showed a significant decrease in pain (from 42 to 14 ) and disability (from 69 to 24) during the whole follow-up period.

\section{Discussion}

Pulsed radiofrequency is a good treatment option for shoulder pain in patients with no surgical indications (8- 


\begin{tabular}{|c|c|c|c|c|c|c|c|}
\hline VAS & Basal & 15 Days & One Month & Three Months & Six Months & 12 Months & 18 Months \\
\hline Resting & 5 & 3 & 3 & 3 & 3 & 3 & 3 \\
\hline Movement & 9 & 4 & 3 & 4 & 2 & 4 & 4 \\
\hline Sleep & 8 & 3 & 3 & 2 & 2 & 2 & 3 \\
\hline SPADI & Basal & 15 Days & One Month & Three Months & Six Months & 12 Months & 18 Months \\
\hline Pain & 42 & 17 & 14 & 13 & 15 & 12 & 14 \\
\hline Disability & 69 & 25 & 26 & 23 & 25 & 26 & 24 \\
\hline
\end{tabular}

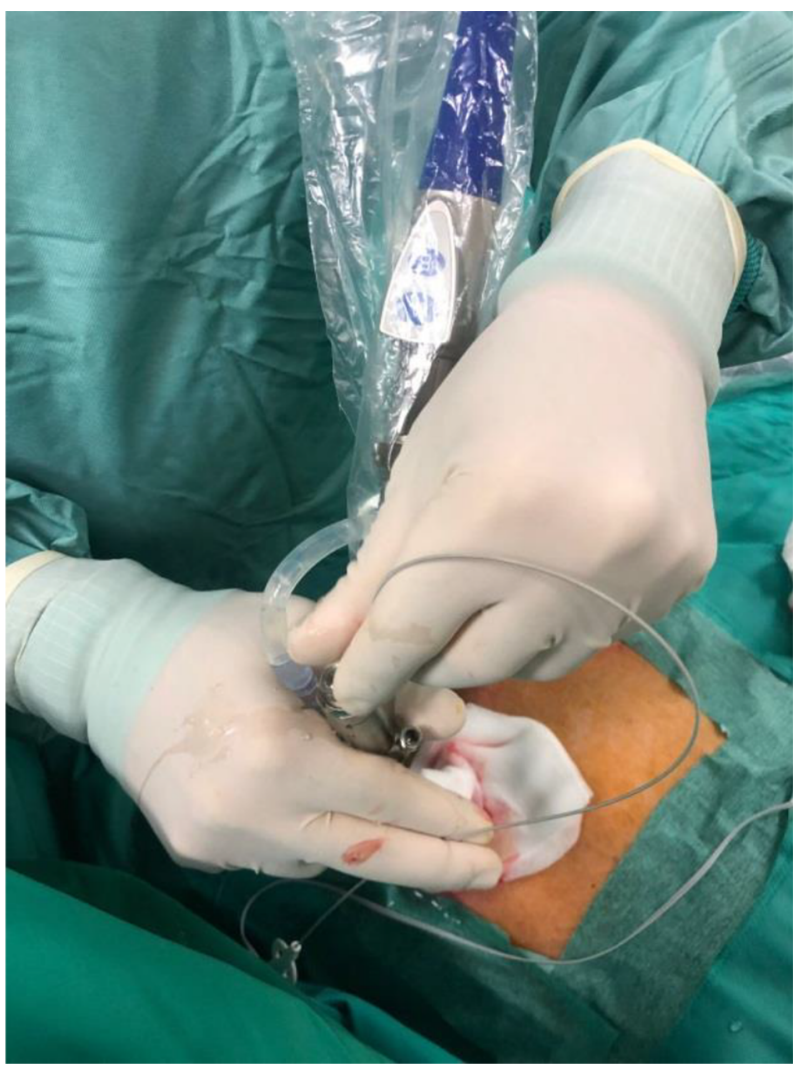

Figure 2. View of the electro catheter inserted in the endoscope for pulsed radiofrequency stimulation of the suprascapular nerve

10). Its long-term efficacy is still not clear and in any case, it does not last longer than 12 - 18 months $(5,7)$. Pharmacological treatment is not always efficacious and may have side effects, especially in long-term treatment, even in the case of prescribing NSAIDs with long-lasting clini-

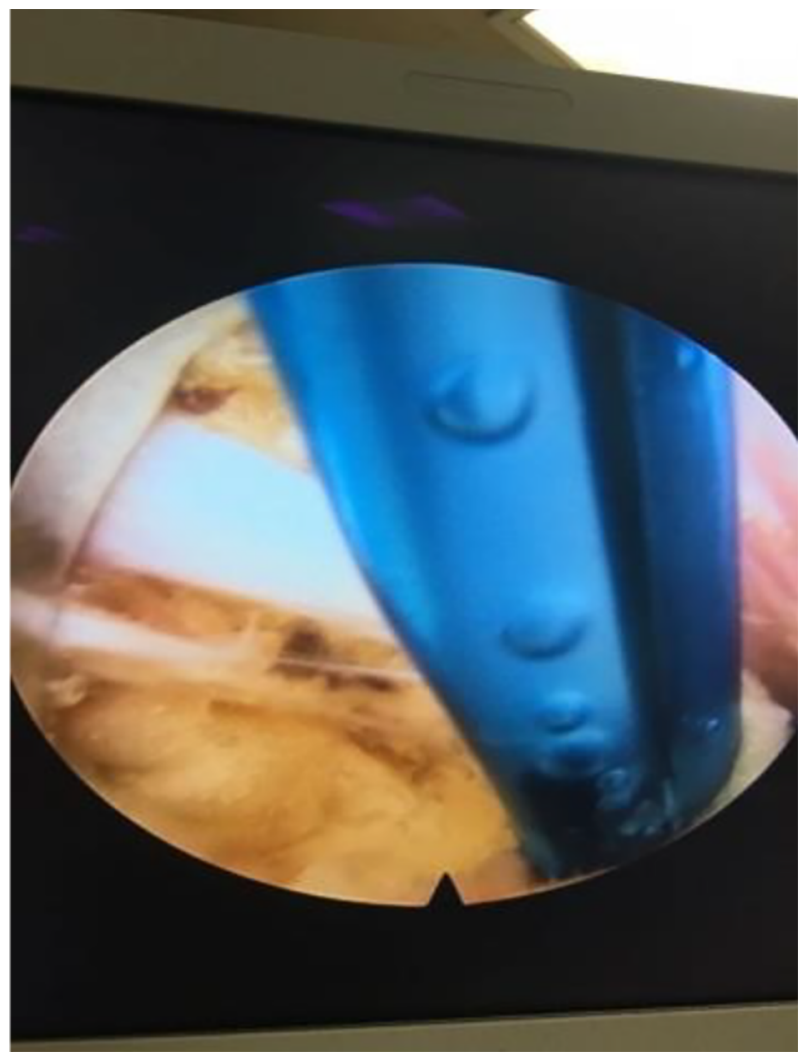

Figure 3. Endoscopic view of the suprascapular nerve (white stripe) and detection of the nerve by the catheter. By endoscopic access, it is possible to have a very clear view of the point of contact of the nerve with the tip of the catheter.

cal use and good safety profile (11-13). Physiotherapy seems somewhat interesting, as much as educational programs are presented, especially for working adults $(14,15)$.

The use of endoscopy to detect the nerve trunk can be a good option to increase the positive results of PRF. This case 
report should be followed by a larger number of cases, possibly in a randomized clinical trial. In this case report, the patient showed very good and stable results for 18 months in terms of pain reduction and shoulder functionality. The technique requires a good knowledge of anatomical landmarks and endoscopic instruments; in our opinion, it can be particularly indicated in patients that had good pain relief with percutaneous PRF in need of repeated procedures.

\section{Footnotes}

Authors' Contribution: Marco Divizia, Giorgio Germani, Ignazio Urti, and Stefano Meloncelli performed the case. Stefano Meloncelli and Marco Divizia drafted the manuscript. Farnad Imani and Giustino Varrassi reviewed and finalized the manuscript. All the authors have approved the final version submitted to this journal.

Conflict of Interests: None of the authors has any conflict of interest.

Ethical Approval: Considering the routine management of the case, Ethics Committee approval was not necessary.

Funding/Support: No funding was received for this study. Informed Consent: The patient was informed and he signed the informed consent form.

\section{References}

1. Picavet HS, Schouten JS. Musculoskeletal pain in the Netherlands: prevalences, consequences and risk groups, the DMC(3)-study. Pain. 2003;102(1-2):167-78. doi: 10.1016/s0304-3959(02)00372-x. [PubMed: 12620608].

2. Malik KM, Beckerly R, Imani F. Musculoskeletal disorders a universal source of pain and disability misunderstood and mismanaged: A critical analysis based on the U.S. model of care. Anesth Pain Med. 2018;8(6). e85532. doi: 10.5812/aapm.85532. [PubMed: 30775292]. [PubMed Central: PMC6348332].

3. Fusco M, Skaper SD, Coaccioli S, Varrassi G, Paladini A. Degenerative joint diseases and neuroinflammation. Pain Pract. 2017;17(4):522-32. doi: 10.1111/papr.12551. [PubMed: 28039964].
4. Neer C2. Anterior acromioplasty for the chronic impingement syndrome in the shoulder.1972. J Bone Joint Surg Am. 2005;87(6):1399. doi 10.2106/JBJS.8706.cl. [PubMed: 15930554]

5. Liliang PC, Lu K, Liang CL, Tsai YD, Hsieh $\mathrm{CH}$, Chen HJ. Pulsed radiofrequency lesioning of the suprascapular nerve for chronic shoulder pain: A preliminary report. Pain Med. 2009;10(1):70-5. doi: 10.1111/j.1526-4637.2008.00543.x. [PubMed: 19222771].

6. Imani F. Using pulsed radiofrequency for chronic pain. Anesth Pain Med. 2012;1(3):155-6. doi: 10.5812/kowsar.22287523.4047. [PubMed: 24904784]. [PubMed Central: PMC4018683].

7. Keskinbora K, Aydinli I. [Long-term results of suprascapular pulsed radiofrequency in chronic shoulder pain].Agri.2009;21(1):16-21. Turkish. [PubMed: 19357996].

8. Chua NH, Vissers KC, Sluijter ME. Pulsed radiofrequency treatment in interventional pain management: Mechanisms and potential indications-a review. Acta Neurochir(Wien). 2011;153(4):763-71. doi: 10.1007/s00701-010-0881-5. [PubMed: 21116663]. [PubMed Central: PMC3059755].

9. Sluijter ME, Imani F. Evolution and mode of action of pulsed radiofrequency. Anesth Pain Med. 2013;2(4):139-41. doi: 10.5812/aapm.10213. [PubMed: 24223349]. [PubMed Central: PMC3821144].

10. Esparza-Minana JM, Mazzinari G. Adaptation of an ultrasound-guided technique for pulsed radiofrequency on axillary and suprascapular nerves in the treatment of shoulder pain. Pain Med. 2019;20(8):154750. doi: $10.1093 / \mathrm{pm} /$ pny311. [PubMed: 30690499].

11. Doiron-Cadrin P, Lafrance S, Saulnier M, Cournoyer E, Roy JS, Dyer JO, et al. Shoulder rotator cuff disorders: A systematic review of clinical practice guidelines and semantic analyses of recommendations. Arch Phys Med Rehabil. 2020. doi: 10.1016/j.apmr.2019.12.017. [PubMed: 32007452].

12. Imani F, Varrassi G. Ketamine as adjuvant for acute pain management. Anesth Pain Med. 2019;9(6). e100178. doi: 10.5812/aapm.100178. [PubMed: 32280623]. [PubMed Central: PMC7119219].

13. Varrassi G, Pergolizzi JV, Dowling P, Paladini A. Ibuprofen safety at the golden anniversary: Are all NSAIDs the same? A narrative review. Adv Ther. 2020;37(1):61-82. doi: 10.1007/s12325-019-01144-9. [PubMed: 31705437].

14. Giacalone A, Alessandria P, Ruberti E. The physiotherapy intervention for shoulder pain in patients treated for breast cancer: Systematic review. Cureus. 2019;11(12). e6416. doi: 10.7759/cureus.6416. [PubMed: 31988817]. [PubMed Central: PMC6970094].

15. Li Y, Tse MYM. An online pain education program for working adults: Pilot randomized controlled trial. J Med Internet Res. 2020;22(1) e15071. doi: 10.2196/15071. [PubMed: 31934865]. [PubMed Central: PMC6996734]. 